\title{
AUTOPERCEPÇÃO DE SAÚDE DE IDOSAS PRATICANTES DE ATIVIDADES FISICAS E FATORES ASSOCIADOS
}

\author{
Emanuelly Casal Bortoluzzi ${ }^{1}$ \\ Julia Pancotte ${ }^{2}$ \\ Marlene Doring ${ }^{3}$ \\ Daniela Bertol Graeff ${ }^{4}$ \\ Ana Luisa Sant'Anna Alves ${ }^{5}$ \\ Marilene Rodrigues Portella ${ }^{6}$ \\ Helenice de Moura Scortegagna ${ }^{7}$ \\ Bernadete Maria Dalmolin ${ }^{8}$
}

\footnotetext{
1 Graduada em Educação Física - Bacharelado. Mestre em Envelhecimento Humano. Docente no Instituto de Desenvolvimento do Alto Uruguai (IDEAU campus Getúlio Vargas). E-mail: manu_casal@ hotmail.com.

2 Graduada em Fisioterapia. Mestre em Envelhecimento Humano. Facilitadora de Barras de Access Consciousness ${ }^{\circledR}$. E-mail: jupancotte@hotmail.com.

3 Graduada em Enfermagem. Doutora em Saúde Pública. Docente do Programa de Pós-graduação stricto sensu Envelhecimento Humano. E-mail: doring@upf.br.

4 Graduada em Fisioterapia. Mestrado em Epidemiologia. Docente no curso de Medicina na Universidade de Passo Fundo. E-mail: bertoldani@gmail.com.

5 Graduada em Nutrição. Doutora em epidemiologia. Docente no curso de Nutrição na Universidade de Passo Fundo. E-mail: alves.als@gmail.com.

6 Graduada em Enfermagem. Doutora em Enfermagem. Docente do Programa de Pós-graduação stricto sensu Envelhecimento Humano. E-mail: portella@upf.br.

7 Graduada em Enfermagem. Doutora em Enfermagem. Docente do Programa de Pós-graduação stricto sensu Envelhecimento Humano. E-mail: scortegagna@upf.br.

8 Graduada em Enfermagem. Doutora em Saúde Pública. Atualmente é reitora da Universidade de Passo Fundo. E-mail: berna@upf.br.
} 
resumo

O objetivo do estudo foi verificar a autopercepção de saúde de idosas praticantes de atividade física e fatores associados. Foi realizado um estudo transversal, recorte do estudo longitudinal do Centro de Referência e Atenção ao Idoso, no período de 2014 e 2015. Aplicou-se um questionário estruturado com questões sociodemográficas, comportamentais e clínicas. Participaram idosas que realizavam uma ou mais oficinas de exercícios físicos, como alongamento, ioga, pilates no solo, treinamento funcional e dança, no mínimo duas vezes por semana, há pelo menos um mês. Realizou-se análise descritiva e inferencial. Participaram do estudo 293 idosas, com idade média de 69 anos $( \pm 6,9)$. A autopercepção de saúde foi relatada como positiva por 75,4\% das idosas, e com relação a autopercepção de saúde negativa, não houve referência a saúde muito ruim e menos de 1\% para saúde ruim. Na comparação de sua saúde no dia da entrevista com a de um ano atrás, 29,5\% consideraram melhor e $56,8 \%$ igual, comparando com pessoas da mesma idade 62,5\% referem estar melhor e 33,8\% igual. Quanto a doenças crônicas, a maior frequência foi de hipertensão (58,6\%), seguida por arritmia cardíaca (17,1\%) e diabetes (11\%). Houve associação entre autopercepção de saúde negativa e escolaridade, diabetes, arritmia cardíaca e uso de medicação. Conclui-se que a autopercepção de saúde pode ser influenciada pela escolaridade, condições clínicas, adesão e continuidade da prática de exercícios físicos em grupo.

palavras-chave

Envelhecimento. Atividade Física. Saúde da Mulher. Autopercepção.

No Brasil, o envelhecimento populacional ocorre pelo aumento da expectativa de vida, e isso se deve a maiores investimentos nas medidas de saúde pública se comparado a décadas anteriores. A taxa de crescimento da população idosa é de mais de $4 \%$ ao ano no período de 2012 a 2022, sendo que a população idosa pode chegar a 41,5 milhões em 2030 e 73,5 milhões em 2060 (IBGE, 2015). Diante dessa perspectiva, surgem estudos sobre o envelhecimento saudável, 
que pode ser definido como o processo de desenvolvimento e manutenção da capacidade funcional que permite o bem-estar em idade avançada (OMS, 2015).

A autopercepção de saúde é um conceito multidimensional, que contempla aspectos de dimensão física, cognitiva e emocional, fatores sociodemográficos e de relações sociais, assim como o bem-estar geral do indivíduo (SIMON et al., 2005; NUNES; BARRETO; GONÇALVES, 2012). Assim, é considerada uma forma confiável de avaliação do estado de saúde geral, além de relevante preditor de mortalidade em todas as idades, em especial nos idosos (GILTAY; VOLLAARD; KROMHOUT, 2011; CARDOSO et al., 2014).

Nesse sentido, valores de autopercepção de saúde ruim ou muito ruim maiores do que $25 \%$ exigem uma avaliação por parte dos profissionais de saúde (PAGOTTO; BACHION; SILVEIRA, 2013). Esse indicador de saúde prático e econômico deve ser considerado pelos profissionais e por políticas públicas, para que os comprometimentos que prejudicam, de forma mais veemente, a qualidade de vida desses idosos sejam postergados (PILGER; MENON; MATHIAS, 2011).

Com relação às idosas, estas aparecem como maioria nos estudos (CARDOSO et al., 2014; PASKULIN; VIANNA, 2007; BORGES et al., 2014; CAMPOS et al., 2015), provavelmente devido ao fenômeno de feminização do envelhecimento, que ocorre pela maior expectativa de vida das mulheres. Ainda, essas referem com maior frequência autopercepção negativa de saúde em comparação aos homens (CONFORTIN et al., 2015).

É necessário ressaltar que a prática de exercícios físicos regulares é um fator comportamental que auxilia na prevenção e tratamento de doenças crônicas, além de estar associada a melhor qualidade de vida (OMS, 2005). Porém, a inatividade física é comum, principalmente entre os idosos (MACEDO et al., 2016). E, quando se pensa nos idosos, que pode ser considerada uma população especial, o exercício físico, com vista a um estilo de vida ativo para um envelhecimento bem sucedido, pode ser oferecido por meio de diferentes modalidades e intervenções como uma das estratégias eficazes para aumentar a expectativa de vida ativa (MAZO, 2008).

A realização de exercícios físicos regulares e moderados pode retardar declínios funcionais, ajudando pessoas idosas a ficarem tão independentes quanto possível na longevidade, além de reduzir o risco de quedas (MAZO, 2008). Por isso, há benefícios economicamente importantes quando os idosos são fisicamente ativos, com custos médicos menores, incluindo menor necessidade de intervenções de saúde onerosas ao Estado, tais como: medicações, internações hospitalares, consultas médicas, dentre outras (OMS, 2005; PASKULIN; VIANNA, 2007; CHO, 2014). 
Por essa razão, a investigação com a população citada, na condição de participantes de um grupo de convivência e prática de exercícios físicos, pode elucidar os fatores associados a autopercepção positiva e negativa de saúde. Logo, este estudo tem como objetivo verificar a autopercepção de saúde de idosas praticantes de atividade física e os fatores associados.

\section{Metodologia}

Trata-se de estudo transversal, recorte de um estudo longitudinal do Centro de Referência e Atenção ao Idoso da Universidade de Passo Fundo/RS: ELO-Creati. Todos os idosos matriculados no período de 2014 e 2015 foram convidados a participar da pesquisa. A coleta de dados foi realizada individualmente, em horário previamente agendado e por meio da aplicação de um questionário estruturado.

O referido centro de referência, vinculado a uma Universidade Comunitária, oferece serviços aos idosos do município há 27 anos, oferecendo oficinas e atividades socioculturais, educacionais e físicas. Funciona de segunda a sexta-feira, nos turnos da manhã e da tarde, tendo a primeira atividade às 8 horas e a última finalizando às 18 horas. Nas oficinas, os idosos participam de turmas, com horários fixos e definidos, uma a duas vezes por semana, para fins de ter materiais e espaço suficientes ao tamanho das turmas, prezando a qualidade das mesmas. Atividades extras são abertas à comunidade, sem restrição de quantidade e oferecidas aleatoriamente durante todo o ano. Todas essas modalidades são oferecidas por instrutores qualificados, com formação em diversas áreas, e também por professores da referida Universidade.

Para este estudo, foram adotados como critérios de inclusão ser do sexo feminino, com idade acima de 60 anos e realizar pelo menos uma oficina de exercícios físicos no mínimo duas vezes por semana, há pelo menos um mês. As idosas pertenciam às seguintes oficinas: alongamento, ioga, pilates no solo, exercício funcional ou dança; sendo que a maioria realizava apenas uma dessas modalidades, e, algumas idosas, duas oficinas. O tempo de participação das idosas nas diferentes oficinas de exercícios físicos variou de menos de um ano até 25 anos.

As variáveis investigadas foram idade, estado civil (com companheiro/ sem companheiro), se residia sozinha (sim/não), escolaridade, tabagismo (sim/ não), etilismo (sim/não), uso de medicação (sim/não), tempo de participação no grupo de convivência (meses), autopercepção de saúde (muito boa, boa, regular, ruim e muito ruim) e presença de morbidades, como: hipertensão arterial, diabetes mellitus, acidente vascular cerebral, infarto agudo do miocárdio, insuficiência cardíaca e arritmia cardíaca. 
A variável autopercepção de saúde contempla três questões: autopercepção da própria saúde; comparação com um ano atrás; e comparação com outras pessoas da sua idade. Considerou-se autopercepção de saúde positiva as respostas muito boa ou boa, e negativa para as respostas regular, ruim ou muito ruim.

Foram realizadas análises descritivas e inferenciais no pacote estatístico SPSS versão 18.0. Para verificar a associação entre as variáveis categóricas, foram aplicados o Teste Qui-quadrado e o Teste Exato de Fisher. O nível de significância considerado foi de $5 \%$.

O projeto foi aprovado pelo Comitê de Ética em Pesquisa da Universidade de Passo Fundo sob parecer n. ${ }^{-}$741.214. Todos os participantes da pesquisa foram preservados através do termo de consentimento livre e esclarecido assinado pelo pesquisador e pelo participante da pesquisa.

\section{Resultados}

Participaram do estudo 293 idosas, com idade entre 60 e 89 anos $(69,35 \pm 6,9)$. Entre as idosas que avaliaram sua saúde positivamente, a média de idade foi de 68,94 $\pm 6,9$ (IC 95\% = 68,02 - 69,85); já entre as que tiveram autopercepção de saúde negativa, a média foi de 70,62 $\pm 6,8$ (IC $95 \%=69,01-72,24$ ). A maioria das idosas avaliou positivamente sua saúde $(75,4 \%)$, ou seja, classificaram sua saúde como muito boa ou boa (Tabela 1).

Tabela 1 - Prevalência de autopercepção de saúde positiva e negativa em proporções e intervalo de confiança, Passo Fundo, 2014 - 2015.

\begin{tabular}{lll}
\hline & $\mathbf{n}(\%)$ & IC 95\% \\
\hline $\begin{array}{l}\text { Autopercepção } \\
\text { de saúde positiva }\end{array}$ & $221(75,4)$ & $71,0-80,2$ \\
$\begin{array}{l}\text { Autopercepção } \\
\text { de saúde negativa }\end{array}$ & $72(24,6)$ & $19,8-29,0$ \\
Autopercepção de saúde & & \\
Muito boa & $56(19,1)$ & $14,7-23,9$ \\
Boa & $165(56,3)$ & $50,2-62,1$ \\
Regular & $70(23,9)$ & $19,1-28,7$ \\
Ruim & $2(0,7)$ & $0,0-1,7$ \\
\hline
\end{tabular}

Fonte: Elaborada pelos autores. 
Quando as idosas foram solicitadas a comparar sua saúde de hoje (dia da entrevista) com sua saúde de um ano atrás, 29,5\% consideraram melhor, $56,8 \%$ igual e 13,7\% pior. Com relação à comparação de sua saúde com outras pessoas da mesma idade, $62,5 \%$ referiram estar melhor, $33,8 \%$ igual e $3,1 \%$ pior. As variáveis escolaridade e uso de medicamentos mostraram-se associadas à autopercepção de saúde, os resultados sugerem que a maior escolaridade está associada à autopercepção positiva da saúde (Tabela 2).

Tabela 2 - Associação entre autopercepção de saúde e características sociodemográficas, comportamentais e uso de medicação, Passo Fundo, 2014 - 2015.

\begin{tabular}{|c|c|c|c|}
\hline \multirow{3}{*}{ Características } & \multicolumn{2}{|c|}{ Autopercepção de saúde } & \multirow{3}{*}{$p^{*}$} \\
\hline & \multirow{2}{*}{$\begin{array}{l}\text { Positiva } \\
\mathrm{n}(\%)\end{array}$} & \multirow{2}{*}{$\begin{array}{l}\text { Negativa } \\
\mathrm{n}(\%) \\
\end{array}$} & \\
\hline & & & \\
\hline \multicolumn{4}{|c|}{ Possui companheiro $^{+}$} \\
\hline Sim & $82(78,1)$ & $23(21,9)$ & \multirow[t]{2}{*}{0,259} \\
\hline Não & $139(73,9)$ & $49(26,1)$ & \\
\hline \multicolumn{4}{|l|}{ Reside sozinha $^{\dagger \dagger}$} \\
\hline Sim & $79(72,5)$ & $30(27,5)$ & \multirow[t]{2}{*}{0,710} \\
\hline Não & $140(76,9)$ & $42(23,1)$ & \\
\hline \multicolumn{4}{|l|}{ Escolaridade } \\
\hline O a 4 anos & $25(67,6)$ & $12(32,4)$ & \multirow[t]{4}{*}{0,049} \\
\hline 5 a 8 anos & $55(67,9)$ & $26(32,1)$ & \\
\hline 9 a 12 anos & $37(74)$ & $13(26)$ & \\
\hline 13 ou mais & $104(83,2)$ & $21(16,8)$ & \\
\hline \multicolumn{4}{|l|}{ Tabagistas } \\
\hline Sim & $6(54,5)$ & $5(45,5)$ & \multirow[t]{3}{*}{0,257} \\
\hline Não & $169(76,5)$ & $52(23,5)$ & \\
\hline Parei de fumar & $46(75,4)$ & $15(24,6)$ & \\
\hline \multicolumn{4}{|l|}{ Etilistas ${ }^{\dagger+t}$} \\
\hline Sim & $36(90)$ & $4(10)$ & \multirow[t]{2}{*}{0,058} \\
\hline Não & $184(73)$ & $68(27)$ & \\
\hline \multicolumn{4}{|l|}{ Uso de medicação } \\
\hline $\operatorname{Sim}$ & $203(74,1)$ & $71(25,9)$ & \multirow[t]{2}{*}{$0,030^{\star \star}$} \\
\hline Não & $18(94,7)$ & $1(5,3)$ & \\
\hline
\end{tabular}

Fonte: Elaborada pelos autores.

* Teste Qui-quadrado. ** Teste Exato de Fisher. † Casada ou união estável. ${ }^{+\dagger}$ Duas participantes

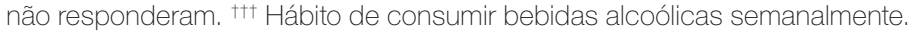


Já entre as doenças crônicas, a prevalência foi de hipertensão arterial sistêmica, presente em $58,6 \%$ das idosas, seguida por arritmia cardíaca (17,1\%) e diabetes mellitus (11\%). A associação mostrou-se significativa entre autopercepção de saúde negativa com a presença de diabetes mellitus $(\mathrm{p}=0,010)$ e arritmia cardíaca $(\mathrm{p}=0,033)$ (Tabela 3).

Tabela 3 - Presença de morbidades e sua associação com autopercepção de saúde, Passo Fundo, $2014-2015$.

\begin{tabular}{|c|c|c|c|}
\hline \multirow{3}{*}{ Morbidades } & \multicolumn{2}{|c|}{ Autopercepção de saúde } & \multirow{3}{*}{$\mathrm{p}^{*}$} \\
\hline & \multirow{2}{*}{$\begin{array}{l}\text { Positiva } \\
\mathrm{n}(\%)\end{array}$} & \multirow{2}{*}{$\begin{array}{l}\text { Negativa } \\
\mathrm{n}(\%)\end{array}$} & \\
\hline & & & \\
\hline \multicolumn{4}{|c|}{ Hipertensão arterial } \\
\hline Sim & $125(73,1)$ & $46(26,9)$ & 0,179 \\
\hline Não & $95(78,5)$ & $26(21,5)$ & \\
\hline \multicolumn{4}{|c|}{ Diabetes mellitus } \\
\hline Sim & $18(56,2)$ & $14(43,8)$ & 0,010 \\
\hline Não & $202(77,7)$ & $58(22,3)$ & \\
\hline \multicolumn{4}{|l|}{ AVC } \\
\hline Sim & $6(66,7)$ & $3(33,3)$ & $0,387^{\star \star}$ \\
\hline Não & $215(75,7)$ & $69(24,3)$ & \\
\hline \multicolumn{4}{|l|}{ IAM } \\
\hline Sim & $6(75)$ & $2(25)$ & $0,625^{\star \star}$ \\
\hline Não & $215(75,4)$ & $70(24,6)$ & \\
\hline \multicolumn{4}{|l|}{ IC } \\
\hline Sim & $8(66,7)$ & $4(33,3)$ & $0,336^{\star \star}$ \\
\hline Não & $213(75,8)$ & $68(24,2)$ & \\
\hline \multicolumn{4}{|c|}{ Arritmia cardíaca } \\
\hline Sim & $32(64)$ & $18(36)$ & 0,033 \\
\hline Não & $189(77,8)$ & $54(22,2)$ & \\
\hline
\end{tabular}

Fonte: Elaborada pelos autores.

* Teste Qui-quadrado. ** Teste Exato de Fisher. AVC: Acidente Vascular Cerebral. IAM: Infarto Agudo do Miocárdio. IC: Insuficiência Cardíaca.

Observa-se diferenças nominais entre tempo de participação nas oficinas e autopercepção de saúde, sendo a autopercepção positiva mais prevalente na categoria de 3 a 8 anos de participação; no entanto, não houve associação significativa entre as variáveis (Tabela 4). 
Tabela 4 - Tempo de participação das idosas no Creati em quartis e sua associação com autopercepção de saúde demonstrados como frequência e percentual [n(\%)], Passo Fundo, $2014-2015$.

\begin{tabular}{|c|c|c|c|c|}
\hline \multirow{3}{*}{$\begin{array}{l}\text { Tempo de } \\
\text { participação }\end{array}$} & \multicolumn{2}{|c|}{ Autopercepção de saúde } & \multirow{3}{*}{$\begin{array}{l}\text { Total } \\
n=291\end{array}$} & \multirow{3}{*}{$\mathrm{p}^{*}$} \\
\hline & Positiva & Negativa & & \\
\hline & $n=219$ & $\mathrm{n}=72$ & & \\
\hline 0 a 2 anos & $47(71,2)$ & $19(28,8)$ & $66(22,7)$ & \\
\hline 3 a 8 anos & $64(79)$ & $17(21)$ & $81(27,8)$ & \\
\hline 9 a 14 anos & $54(77,1)$ & $16(22,9)$ & $70(24,1)$ & \\
\hline 15 a 25 anos & $54(73)$ & $20(27)$ & $54(25,4)$ & 0,674 \\
\hline
\end{tabular}

Fonte: Elaborada pelos autores.

* Teste Qui-quadrado.

\section{Discussão}

O estudo permitiu verificar a elevada prevalência de autopercepção de saúde positiva de idosas praticantes de atividade física em um grupo de convivência e os fatores associados. No entanto, as interpretações devem ser avaliadas com cautela, uma vez que se trata de estudo transversal e há possibilidade de causalidade reversa; a amostra foi por conveniência e pode ter ocorrido o viés de memória. Apesar das limitações, os resultados podem ser úteis para aperfeiçoamento das atividades desenvolvidas no Creati e em outros com amostra similar.

Mulheres, especialmente as idosas, praticam atividades e exercícios físicos em menor proporção do que os homens (PASKULIN; VIANNA, 2007; MAZO, 2008; CAMPOS et al., 2015). Porém, quando estas realizam exercícios físicos, os resultados relacionados à autopercepção de saúde mostram-se positivos. Essa influência pode ocorrer devido à prática regular de exercícios físicos, diminuindo os efeitos dos distúrbios causados pelo período da menopausa, em que as mulheres sofrem diminuição de massa magra e aumento da gordura corporal, fatores que acentuam o risco para doenças crônicas e declínio funcional (SIMS et al., 2013; FORTALEZA et al., 2014).

Em outro estudo realizado no município de Passo Fundo/RS, em que foram entrevistados idosos de ambos os gêneros em vários pontos do município, dos quais cerca de $67 \%$ relataram praticar atividades físicas, os valores foram menores em relação à autopercepção positiva e maiores quanto à autopercepção negativa (BORGES et al., 2014). Do mesmo modo, Cardoso et al. 
(2014) e Rocca et al. (2015), em estudos de base populacional realizados em diferentes locais, apresentaram idosas com maior proporção de autopercepção negativa de saúde. Destaca-se que Cardoso et al. (2014) não avaliaram a prática de atividade física e Rocca et al. (2015) concluíram que havia associação entre a prática de atividade física e melhores condições de saúde, tais como menor utilização de serviços de saúde e melhor autoavaliação de saúde.

Nesse sentido, partindo dos achados na comunidade, nos estudos populacionais, em especial no mesmo município (BORGES et al., 2014), os resultados do nosso estudo, com participantes de um grupo de convivência para idosos, demonstraram a relevância da prática de exercícios físicos nesses grupos específicos, visto que esses fatores contribuem no aspecto físico, emocional, psicológico e social (RIGO; TEIXEIRA, 2005; SILVA; BARROS, 2012). Ao analisar a autopercepção de saúde como indicador multidimensional, sugere-se que os benefícios nos diversos aspectos possibilitam os resultados positivos do presente estudo.

Contudo, quanto à autopercepção de saúde negativa, pode-se atribuí-la à dependência funcional no idoso (SILVA et al., 2012), gerando assim a hipótese que idosos que percebem sua saúde ruim ou muito ruim não possuem condições físicas e motivação para participar de grupos de convivência e/ou exercícios, concordando com Borges et al. (2014) ao apresentar maior número de idosos da comunidade com autopercepção negativa.

Quanto às classificações de autopercepção de saúde positiva e negativa, a condição "regular" é incluída por vezes como positiva (NUNES; BARRETO; GONÇALVES, 2012; HU; LU, 2015) e por vezes negativa (CONFORTIN et al., 2015; ALVES; RODRIGUES, 2005; LEE; PARK; KIM, 2015). No presente estudo, optou-se por incluir como negativa; no entanto, ressalta-se que não houve nenhuma indicação de saúde muito ruim e menos de 1\% para saúde ruim, resultados estes inferiores à literatura (ROCHA; FREIRE, 2007; BORGES et al., 2014; CHO, 2014; ROCCA et al., 2015).

Ainda no que concerne à comparação da própria saúde com a de outras pessoas da mesma idade, apenas 3,1\% das idosas considerou sua saúde pior, número inferior à literatura para populações de mulheres idosas: 29,3\%, encontrado por Rocha e Freire (2007) e 13\%, relatado por Tavares et al. (2013). O fato de as idosas da nossa amostra participarem de um grupo de convivência e praticarem exercícios físicos favorece um estilo de vida saudável, o que também pode ser percebido pelo baixo número de pessoas tabagistas e que consomem bebidas alcoólicas semanalmente.

A associação entre autopercepção de saúde positiva e maior escolaridade, encontrada em nosso estudo, aponta para a influência das condições econômicas das idosas, visto que, de maneira geral, maior escolaridade denota maior 
nível socioeconômico, além disso, essas possuem maior nível de informação sobre os cuidados necessários para a saúde. Dessa forma, maior escolaridade pode ser considerada fator de proteção para autopercepção de saúde negativa (ALVES; RODRIGUES, 2005).

Embora não apresente associação, percebe-se que as idosas que possuem companheiro e não moram sozinhas apresentam proporção maior de autopercepção positiva de saúde do que as que não possuem companheiro e moram sozinhas, fator esse que demonstra a magnitude das relações sociais e familiares na autopercepção de saúde. Além disso, morar só é mais um efeito do rápido envelhecimento populacional, em especial do maior número de idosas (PASKULIN; VIANNA, 2007).

Quanto ao uso de medicação, neste estudo foi possível perceber uma alta prevalência de idosas sob uso de medicação, além de mostrar associação com a autopercepção de saúde negativa, corroborando com resultados encontrados também em outros estudos (PASKULIN; VIANNA, 2007; CARDOSO et al., 2014; SILVA; BARROS, 2012; TAVARES et al., 2013). Silva et al. (2012) consideram que o uso contínuo de medicamentos por si só já indica presença de morbidades, e, quando somado às dificuldades de acesso e de controle no uso diário, pode contribuir para uma autopercepção menos otimista da saúde. A autopercepção de saúde negativa também se mostrou associada à presença de diabetes mellitus e arritmia cardíaca, onde observamos resultados semelhantes ao estudo de $\mathrm{Hu}$ e Lu (2015), que encontrou associação significativa entre diabetes mellitus $(\mathrm{OR}=2,63)$, doenças cardíacas $(\mathrm{OR}=1,72)$, prática de exercícios físicos 5 vezes por semana $(\mathrm{OR}=0,56)$ e autopercepção negativa.

Mais de 75\% das idosas participam deste Centro há mais de dois anos, o que mostra a adesão e a manutenção da prática de exercícios físicos, o que pode explicar os valores de autopercepção de saúde positiva. Logo, pressupõe-se que a realização de exercício físico e de forma coletiva pode influenciar positivamente na autopercepção de saúde, bem como não participar pode exercer influência negativa.

\section{Conclusão}

A autopercepção de saúde positiva das idosas mostrou-se elevada na amostra investigada, sendo associada a maior escolaridade, não utilizar medicamentos e não apresentar diabetes mellitus e arritmia cardíaca. De acordo com a literatura, um dos fatores que pode ter contribuído para a elevada prevalência de autopercepção positiva de saúde é a prática de exercícios físicos, pois este hábito auxilia na manutenção do bem-estar geral do indivíduo. Diante disso, 
pressupõe-se que idosos que participam de grupos de exercícios físicos e de convivência possuem melhor bem-estar geral de saúde, se comparados a idosos da comunidade em geral.

Os achados sinalizam a relevância e potencial dos grupos de convivência de idosos na contribuição para a melhoria das condições de saúde. Entretanto, estudos longitudinais são pertinentes para esclarecer as hipóteses aqui apresentadas.

\author{
SELF-PERCEPTION OF HEALTH OF ELDERLY \\ WOMEN PRACTICERS OF PHYSICAL \\ ACTIVITIES AND ASSOCIATED FACTORS
}

\title{
abstract
}

This study aimed to verify the self-perception of health of elderly women engaged in physical activities, and associated factors. A cross-sectional study was performed as a cutout from the longitudinal study from Referral and Care Center to the Elderly: ELO-Creati, from 2014 to 2015. A questionnaire was applied with sociodemographic, behavioral, and clinical questions. Participating in the study were elderly women engaged in one or more workshops of physical exercises, like stretching, yoga, solo pilates, functional training, and dance, at least twice a week and for at least one month. A descriptive and inferential analysis was performed. Participating in the study were 293 elderly women with average age of 69 years $( \pm 6.9)$. The self-perception of health was reported as positive by $75.4 \%$ of the elderly women and there was no reference to very poor health. When comparing their health on the day of the interview and one year previous, 29.5\% considered it better and 56.8\% considered it the same. When comparing with people of the same age, $62.5 \%$ claimed to feel better and 33.8\% claimed to feel the same. As for chronic diseases, the higher frequency was for hypertension (58.6\%), followed by cardiac arrhythmia (17.1\%), and diabetes (11\%). The time of participation in the group varied from one month up to 25 years. There was association between negative self-perception of health and level of education, diabetes mellitus, cardiac arrhythmia, and use of medication. It was concluded that self-perception of health may be influenced by level of education, clinical conditions, engagement and continuity of group physical exercises.

keywords

Aging. Physical Activity. Senior Centers. Women's Health. Self Concept. 
ALVES, Luciana; RODRIGUES, Roberto. Determinantes da autopercepção de saúde entre idosos do Município de São Paulo, Brasil. Revista Panamericana de Salud Pública = Pan American Journal of Public Health, Washington, D.C., v. 17, n. 5/6, p. 333-341, 2005.

BORGES, Aline et al. Autopercepção de saúde em idosos residentes em um município do interior do Rio Grande do Sul. Revista Brasileira de Geriatria e Gerontologia, Rio de Janeiro, v. 17, n. 1, p. 79-86, 2014.

CAMPOS, Ana Cristina et al. Gender differences in predictors of self-rated health among older adults in Brazil and Chile. BMC Public Health, London, v. 15, n. 1, p. 365-376, 2015.

CARDOSO, Joana et al. Poor self-rated health and associated factors among elderly urban residents. Revista Gaúcha de Enfermagem, Porto Alegre, v. 35, n. 4, p. 35-41, 2014.

$\mathrm{CHO}$, Kang-OK. The positive effect of physical activity on health and health-related quality of life in elderly Korean people: evidence from the Fifth Korea National Health and Nutrition Examination Survey. Journal of Lifestyle Medicine, Chesterfield, v. 4, n. 2, p. 86-94, 2014

CONFORTIN, Susana et al. Autopercepção positiva de saúde em idosos: estudo populacional no Sul do Brasil. Cadernos de Saúde Pública, Rio de Janeiro, v. 31, n. 5, p. 1049-1060, 2015

FORTALEZA, Ana Claudia et al. Gordura corporal total e do tronco e o desempenho da marcha em mulheres na menopausa. Revista Brasileira de Ginecologia e Obstetrícia, São Paulo, v. 36, n. 4, p. 176-181, 2014.

GILTAY, Erik; VOLLAARD, Albert; KROMHOUT, Daan. Self-rated health and physician-rated health as independent predictors of mortality in elderly men. Age and ageing, Oxford, v. 41, n. 2, p. 165-171, 2011

HU, Wen; LU, Jiehua. Associations of chronic conditions, APOE4 allele, stress factors, and health behaviors with self-rated health. BMC Geriatrics, London, v. 15, p. 137-145, 2015.

INSTITUTO BRASILEIRO DE GEOGRAFIA E ESTATÍSTICA (IBGE). Mudanças demográficas no Brasil no início do século XXI: subsídios para as projeções da população. Rio de Janeiro: IBGE, 2015

LEE, Jung-A; PARK, Jong Heon; KIM, Myung Kim. Social and physical environments and self-rated health in urban and rural communities in Korea. International Journal of Environmental Research and Public Health, Basel, v. 12, n. 11, p. 14329-14341, 2015.

MACEDO, Rafael et al. Nível de atividade física de idosos participantes de um programa de prevenção de doença cardiovascular. ASSOBRAFIR Ciência, São Paulo, v. 6, n. 3, p. 11-20, 2016.

MAZO, Giovana Zarpellon. Atividade física, qualidade de vida e envelhecimento. Porto Alegre: Sulina, 2008.

NUNES, Ana Paula; BARRETO, Sandhi Maria; GONÇALVES, Luana. Relações sociais e autopercepção da saúde: Projeto Envelhecimento e Saúde. Revista Brasileira de Epidemiologia, São Paulo, v. 15, n. 2, p. 415-428, 2012.

ORGANIZAÇÃO MUNDIAL DA SAÚDE (OMS). Envelhecimento ativo: uma política de saúde. Brasília: OMS, 2005.

. Resumo: relatório mundial de envelhecimento e saúde. Genebra: OMS, 2015.

PAGOTTO, Valéria; BACHION, Maria Márcia; SILVEIRA, Erika. Autoavaliação da saúde por idosos brasileiros: revisão sistemática da literatura. Revista Panamericana de Salud Pública $=$ Pan American Journal of Public Health, Washington, D.C., v. 33, n. 4, p. 302-310, 2013. 
PASKULIN, Lisiane; VIANNA, Lucila. Perfil sociodemográfico e condições de saúde auto-referidas de idosos de Porto Alegre. Revista de Saúde Pública, São Paulo, v. 41, n. 5, p. 757-768, 2007.

PILGER, Calíope; MENON, Mario Humberto; MATHIAS, Thais. Características sociodemográficas e de saúde de idosos: contribuições para os serviços de saúde. Revista Latino-Americana de Enfermagem, Ribeirão Preto, v. 19, n. 5, p. 1230-1238, 2011.

RIGO, Maria de Lourdes; TEIXEIRA, Denílson. Efeitos da atividade física na autopercepção de bem-estar de idosas que residem sozinhas e acompanhadas. UNOPAR Científica: Ciências Biológicas e da Saúde, Londrina, v. 7, n. 1, p. 13-20, 2005.

ROCCA, Patricia et al. Is the association between physical activity and healthcare utilization affected by self-rated health and socio-economic factors? BMC Public Health, London, v. 15, n. 1, p. 737-744, 2015.

ROCHA, Saulo; FREIRE, Malú. Nível de atividade física habitual e autopercepção do estado de saúde em idosas no município de Jequié-Bahia. Revista Brasileira em Promoção da Saúde, Fortaleza, v. 20, n. 3, p. 161-167, 2007.

SILVA, Mariana Poli da; BARROS, Carmen Malaguti de. Benefícios de um programa de exercícios funcionais no tratamento da osteoartrite de joelho. SAÚDE, São Paulo, v. 1 , n. 1, p. 23-42, 2012

SILVA, Roberto Jerônimo et al. Prevalência e fatores associados à autopercepção negativa da saúde em pessoas idosas no Brasil. Revista Brasileira de Epidemiologia, São Paulo, v. 15, n. 1, p. 49-62, 2012.

SIMON, Jeanette et al. How is your health in general? A qualitative study on self-assessed health. The European Journal of Public Health, Stockholm, v. 15, n. 2, p. 200-208, 2005

SIMS, Stacy et al. Changes in physical activity and body composition in postmenopausal women over time. Medicine and Science in Sports and Exercise, Indianapolis, v. 45, n. 8, p. $1486-1492,2013$.

TAVARES, Darlene Mara et al. Comparação das características sociodemográficas de saúde e qualidade de vida de idosos rurais segundo sexo. Revista de Enfermagem e Atenção à Saúde, Uberaba, v. 2, n. 1, p. 32-46, 2013.

Data de Submissão: 09/05/2016

Data de Aprovação: 01/06/2018 